\title{
20 Mathematical modelling of angiogenesis and vascular adaptation ${ }^{1}$
}

\author{
Tomas Alarcon ${ }^{a}$, Helen Byrne ${ }^{b}$, Philip Maini ${ }^{c}$ and Jasmina Panovska ${ }^{c}$ \\ ${ }^{a}$ Bioinformatics Unit, Department of Computer Science, \\ University College London, London, UK \\ ${ }^{b}$ Centre for Mathematical Medicine, Division of Applied \\ Mathematics, University of Nottingham, Nottingham, UK \\ ${ }^{c}$ Centre for Mathematical Biology, Mathematical Institute, \\ University of Oxford, Oxford, UK
}

\section{INTRODUCTION}

Angiogenesis, i.e. the process whereby new blood vessels are formed in order to supply nutrients and/or metabolites to starving tissue, plays a key role in the normal functions of many healthy organisms and also in pathological situations, such as wound healing or cancer.

In the particular case of cancer, the study of tumour-induced angiogenesis was boosted by the research of Folkman and co-workers (Folkman, 1995). While the development of solid tumours is a rather complicated process, it can be assumed to occur in three different stages. During the first stage, solid tumours are avascular, i.e. they comprise a colony of cells that lacks its own blood supply. During this stage, there is typically a maximum, diffusion-limited size to which the tumour can grow (several millimetres) and it does not normally invade the host organ. Thus, avascular tumours are relatively harmless. The second stage of tumour growth starts when some of the cells within the avascular tumour secrete and release so-called tumour-angiogenic factors (TAFs). These substances diffuse towards the nearby vasculature, whereupon they degrade the basal membrane of the blood vessels and promote (chemotactic) migration

\footnotetext{
${ }^{1}$ The authors would like to dedicate this paper to the memory of their friend and colleague, Ray Paton.
} 
towards the source of TAF and proliferation of the endothelial cells (ECs). Once the tumour has thus established its own blood supply, the third phase of vascular tumour growth starts. In this stage, the tumours may become lethal for two main reasons. First, they have access to a virtually endless supply of nutrients and, in consequence, may exhibit the uncontrolled growth typical of cancer. Second, the new vasculature can act as an escape route, enabling the tumour cells to migrate to other parts of the body (metastasis).

Once a solid tumour has successfully established a blood supply, other issues related to blood flow through the vascular network become important in determining the tumour's growth and response to treatment. The major factor here is probably blood flow heterogeneity which is recognised as one of the key physical barriers to drug delivery in tumour treatment (Jain, 2001). Drugs are usually blood-borne, and therefore heterogeneity in blood flow means that most of the injected drug will fail to reach the tumour and be eliminated or, even worse, assimilated by healthy tissue producing deleterious side effects. On the other hand, most nutrients are also blood-borne and therefore heterogeneity in blood flow induces heterogeneity in nutrient distribution within the tissue. This factor and its effects on tumour growth have been recently analysed (Alarcon et al., 2003).

Both angiogenesis and blood flow through vascular networks are active fields of research in the theoretical and mathematical biology communities. In this chapter, we give a brief review of continuum models that have been proposed to describe tumour-induced angiogenesis. Then we present some recent results concerning the organisation of vascular networks and how this is coupled to blood flow and the behaviour of the surrounding tissue. Finally, we present our conclusions.

\section{REVIEW OF CONTINUUM MODELS OF TUMOUR-INDUCED ANGIOGENESIS}

In this section, we describe briefly deterministic models of tumour-induced angiogenesis. Since there are several excellent and detailed reviews in the literature (see for example, Mantzaris et al., 2004, or Preziosi, 2003), we choose not to go into detail here. We simply summarise the general features of the various models and discuss their strengths and weaknesses.

Some of the earliest continuum models of tumour angiogenesis are based on an analogy with fungal growth, both phenomena exhibiting interconnected, branched structures that evolve in response to environmental signals. For example, Balding and McElwain (1985) adapted a 
fungal growth model developed by Edelstein (1982). They focused on three key physical variables: a generic, diffusible chemical produced by the tumour and termed as tumour-angiogenesic factor (or TAF), capillary tips, and capillary sprouts. Their one-dimensional model consisted of three nonlinear partial differential equations that were derived by applying the principle of mass balance to each species. It was assumed that the capillary tips migrate via Chemotaxis towards the source of TAF, i.e. the tumour. New capillary tips emanated from existing vessels and/or tips at rates that increased with increasing TAF levels and were lost as a result of fusion with other tips and/or vessels (i.e. anastomosis). The production of capillary sprouts was assumed to be driven by migration of the capillary tips, the sprout density increasing at a rate that matched the flux of capillary tips - the so-called "snail trail". The TAF was modelled as a diffusible chemical that was produced by the tumour, underwent natural decay, and was consumed by the migrating capillary tips.

Many modifications to Balding and McElwain's model have been developed, in terms of different production and removal terms for the TAF and the capillary tips (see for example, Byrne and Chaplain, 1995; Orme and Chaplain, 1996). However, in each case, the results are similar and exhibit many of the characteristic features of angiogenesis that have been observed in vivo (Muthukkaruppan et al., 1982). Indeed, for parameter values that give rise to successful angiogenesis, typical numerical simulations show an accelerating front of capillary tips and sprouts propagating towards the tumour, the maximum capillary tip density preceding the maximum capillary sprout density.

Recently it has been shown that enhanced migration together with tip production from the vessels via branching, rather than endothelial cell proliferation, can be an alternative to constant migration and additional proliferation of the endothelial cells in the tips for successful angiogenesis (Panovska, 2004). A similar approach has been used before (Anderson and Chaplain, 1998), but without branching, leading to vessels stopping at some distance from the tumour.

Whilst it is straightforward to extend Balding and McElwain' model to two and three space dimensions (Orme and Chaplain, 1997), the resulting models highlight some of the shortcomings of using a continuum framework to study angiogenesis. This is primarily because angiogenesis is a twoor three-dimensional process, with tips sprouting in directions other than that of the propagating vascular front, and it is not immediately clear how the snail trail should be generalised in higher space dimensions. In addition, because the dependent variables are densities, the models are unable to distinguish between different vascular morphologies (e.g. a region perfused by one, large vessel would be identical to a region perfused by many 
small vessels, even though their surface areas and, hence, nutrient delivery rates would differ). Moreover the models do not distinguish between anastomosis and capillary tip death, even though the former will increase nutrient supply to the tissue while the latter will not. Finally, no account is taken of vascular remodelling and, in particular, the impact of blood flow and haematocrit on the evolving vasculature. In spite of these weaknesses, the models do provide useful insights into the ways in which different physical mechanisms (e.g. the strength of the chemotactic response, the rate of TAF production, the rate of capillary tip formation) influence angiogenesis. In particular, the success of angiogenesis is tightly controlled by the balance between endothelial cell proliferation and migration: as the strength of the chemotactic response increases, the tips migrate towards the tumour more rapidly, thereby reducing both the time available for tip proliferation and the density of the vasculature when the tumour is reached.

Anderson and Chaplain (1998) developed a continuum-discrete model in which the movement of the tips of the vessels was modelled by means of a biased random walk whose transition probabilities were derived by discretisation of a previously developed PDE model. In this work, the role of haptotaxis (migration upward gradient of adhesive molecules, in particular fibronectin) was examined and shown to be a key factor in successful angiogenesis.

Since Balding and McElwain's model of tumour angiogenesis was developed, detailed knowledge of the specific chemicals and biochemistry involved has been elucidated. Following Orme and Chaplain (1997), Levine et al. (2001) have developed highly complex models that account for much of this new detail and, as such, these represent an important step towards understanding angiogenesis using biochemically based rather than phenomenological arguments. For example, Levine et al. account for interactions between ECs, angiogenic factors and cells such as pericytes and macrophages that are also involved in angiogenesis. One important difference between Levine et al.'s model and that of Balding and McElwain is their treatment of the ECs cells. Levine et al. based their model on the theory of reinforced random walks developed by Othmer and Stevens (1997).

The models of angiogenesis mentioned above do not account for nutrient delivery to the tissue by the developing vasculature and cease to apply once the capillary tips reach the tumour. Recently, Breward et al. (2003) have used a multiphase modelling framework to develop continuum models of vascular tumour growth that address these shortcomings.

Possibly the main criticism of the above models of angiogenesis stems from their inability to track individual capillary tips and, hence, 
to reproduce accurately the patterns of vascular growth observed during tumour angiogenesis (but see the work of Chaplain and co-workers (Anderson and Chaplain, 1998)). In the next section, we explain how hybrid cellular automata models can be used to resolve these issues.

\section{DESIGN PRINCIPLES AND STRUCTURAL ADAPTATION OF VASCULAR NETWORKS}

In the previous section, we focused on continuum modelling of tumourinduced angiogenesis, explaining how the evolution of the (continuous) density of capillary tips or ECs could be analysed. We now concentrate on interactions between blood flow, signalling cues from the surrounding tissue, and the structure of the vascular tree and show how they may be incorporated into a hybrid cellular automata modelling framework.

\subsection{A design principle for vascular beds: role of complex blood rheology}

The idea that design principles govern the organisation of vascular systems has proved to be a fruitful approach (LaBarbera, 1990). Design principles are based on the hypothesis that the organisation of the vascular system comprises geometrical, physical, and physiological constraints that optimise overall function.

Several design principles based on different hypotheses have been proposed. Gafiychuk and Lubashevsky (2001) based their design principle on the geometrical requirement of space filling. A different approach, based on fractal modelling and scaling concepts, was introduced by Gazit et al. (1995). They measured the fractal dimensions of capillary beds, normal arterial trees, and tumour vasculature. The resulting fractal dimensions were found to be compatible (within experimental errors) with the fractal dimensions of clusters generated by classical, non-equilibrium growth processes extensively studied in statistical mechanics (space filling, diffusion-limited aggregation, and invasion percolation, respectively). The third type of design principle is based on optimisation arguments, whereby some physical quantity (dissipated power (Murray, 1926), wall material (Kurz and Sandau, 1997), etc) is minimised so that the performance of the vascular system is maximised. The best known of these optimisation principles is Murray's law.

Murray's law states that the vascular system is organised so that a balance exists between the metabolic energy of a given volume of blood and the energy required for blood flow. While resistance to blood flow 
diminishes when the radius of the vessel increases, the metabolic energy needed to maintain a larger volume of blood increases. Specifically, Murray's law is based on a minimisation principle for the dissipated power, $W$. The blood is viewed as a Newtonian fluid of constant viscosity and the flow assumed to be Poiseuille. The vessels are considered rigid tubes and the pressure gradient is assumed to be constant. These assumptions lead to the following formulation of the design principle for a single vessel of length $L$ and radius $R$ :

$$
\begin{aligned}
\frac{\partial W}{\partial R} & =0, \\
W & =W_{\mathrm{H}}+W_{\mathrm{M}}, \\
W_{\mathrm{H}} & =\frac{8 Q^{2} \mu_{0} L}{\pi R^{4}}, \\
W_{\mathrm{M}} & =\alpha_{\mathrm{b}} \pi R^{2} L,
\end{aligned}
$$

where $W_{\mathrm{H}}$ is the power dissipated by the flow, $W_{\mathrm{M}}$ is the metabolic energy consumption rate of the blood, $Q$ is the flow rate, $\mu_{0}$ is the blood viscosity, and $\alpha_{\mathrm{b}}$ is the metabolic rate per unit volume of blood. Using Equation (1) we deduce that the total dissipated power $W$ is minimised if

$$
Q=\frac{\pi R^{3}}{4} \sqrt{\frac{\alpha_{\mathrm{b}}}{\mu_{0}}} .
$$

Equation (2) shows how the vessel radius $R$ and the flow rate $Q$ are related when the optimisation principle is satisfied. Additionally, at a bifurcation in the vascular tree, conservation of mass implies:

$$
Q_{\mathrm{P}}=Q_{1}+Q_{2},
$$

where $Q_{\mathrm{P}}$ is the flow rate through the parent vessel, $Q_{1}$ and $Q_{2}$ are the flow rates through the daughter vessels. Taken together, Equations (2) and (3) imply that, at a bifurcation, the radius of the parent vessel, $R_{\mathrm{P}}$, and the radii of the daughter vessels, $R_{1}$ and $R_{2}$, satisfy:

$$
R_{\mathrm{P}}^{3}=R_{1}^{3}+R_{2}^{3} .
$$

This is Murray's basic result concerning the architecture of the vascular tree. Furthermore, using the above result, it is easy to determine the wall shear stress (WSS) within a given vessel. For Poiseuille flow, the WSS is given by

$$
\tau_{\mathrm{w}}=\frac{4 \mu_{0} Q}{\pi R^{3}}=\sqrt{\alpha_{\mathrm{b}} \mu_{0}}
$$


using Equation (2). Thus we conclude that the WSS is constant throughout vascular networks constructed according to Murray's principle.

While the WSS is approximately constant in large arterial vessels, experimental work by Pries et al. (1995) shows that it depends on the transmural pressure in a sigmoidal manner, saturating to a constant value only for large pressures (i.e. large arteries and arterioles).

Murray's law neglects certain factors, such as rheology, which may play important roles in the organisation of the vascular tree. Blood is far from being a simple fluid with constant viscosity: it is a highly complex suspension of cells and molecules of a wide range of sizes. Consequently, treating blood as a Newtonian fluid is a very crude approximation. Red blood cells play a key role in the rheology of blood. The relative (non-dimensional) blood viscosity, $\mu_{\text {rel }}(R, H)$, depends on $R$ and also on the haematocrit, $H$, which is defined as the ratio between the total blood volume and the volume occupied by red blood cells. The relative viscosity exhibits a non-monotonic dependence on $R$, which can be subdivided into three different regions. If $R$ is much greater than the typical size of a red blood cell, then the viscosity is independent of the vessel radius. As the radius of the vessel decreases, the viscosity also decreases (the Fahraeus-Lindqvist effect) until the viscosity attains a minimum. For smaller values of $R$ the viscosity increases as $R$ decreases. The dependence on $H$ is easier to understand: the higher is $H$, the thicker the blood becomes, and therefore its viscosity increases (Pries et al., 1994).

The question we pose concerns whether it is possible to generalise Murray's law in order to obtain results that reproduce more realistically the behaviour of the wall shear stress and, in particular, the wall shear stress-pressure relationship observed by Pries et al. (1995). To this end we reformulate the design principle as follows:

$$
\begin{aligned}
\frac{\partial W}{\partial R} & =0 \\
W & =W_{\mathrm{H}}+W_{\mathrm{M}}, \\
W_{\mathrm{H}} & =\frac{8 Q^{2} \mu_{0} \mu_{\mathrm{rel}}(R, H) L}{\pi R^{4}}, \\
W_{\mathrm{M}} & =\alpha_{\mathrm{b}} \pi R^{2} L
\end{aligned}
$$

which predicts that $Q$ and $R$ are related as follows:

$$
Q=\frac{\pi R^{3}}{4 \mu_{0}} \sqrt{\frac{\alpha_{\mathrm{b}}}{4 \mu_{\mathrm{rel}}-R \partial \mu_{\mathrm{rel}} / \partial R}} .
$$


This equation needs to be solved numerically since the analytical expression for $\mu_{\text {rel }}(R, H)$, i.e.

$$
\begin{aligned}
\mu_{\text {rel }}(R, H) & =\left[1+\left(\mu_{0.45}^{*}-1\right) \frac{(1-H)^{C}-1}{(1-0.45)^{C}-1}\left(\frac{2 R}{2 R-1.1}\right)^{2}\right]\left(\frac{2 R}{2 R-1.1}\right)^{2}, \\
\mu_{0.45}^{*} & =6 e^{-0.17 R}+3.2-2.44 e^{-0.06(2 R)^{0.645}} \\
C & =\left(0.8+e^{-0.15 R}\right)\left(\frac{1}{1+10^{-11}(2 R)^{12}}-1\right)+\frac{1}{1+10^{-11}(2 R)^{12}}
\end{aligned}
$$

(see Pries at al., 1994) is very complicated. Even though its solution is of the same type as that obtained by Murray, i.e. $Q=C(R, H) R^{\alpha(R, H)}$. In the present case, however, the coefficient $C$ and the branching exponent $\alpha$ depend on $R$ and $H$. This fact, together with mass conservation at a bifurcation, leads to the following equation relating the radii of the parent and daughter vessels:

$$
C\left(R_{\mathrm{P}}, H_{\mathrm{P}}\right) R_{\mathrm{P}}^{\alpha\left(R_{\mathrm{P}}, H_{\mathrm{P}}\right)}=C\left(R_{1}, H_{1}\right) R_{1}^{\alpha\left(R_{1}, H_{1}\right)}+C\left(R_{2}, H_{2}\right) R_{2}^{\alpha\left(R_{2}, H_{2}\right)}
$$

(See Alarcon et al., 2005a for full details).

The main difference between Equations (4) and (9) is that the last one is local, in the sense that it depends on the state of the vessels at a particular bifurcation. By contrast, Equation (4) is global. Later, we will see that the local nature of Equation (9) has important implications.

In order to proceed further, we have used our design principle to construct simple branching vascular networks. A full analysis of these simulations is presented in Alarcon et al. (2005a), here we only summarise the main results. Concerning the behaviour of the wall shear stress, we have found that in our optimal branching network it varies with the pressure (see fig. 1a). Indeed, our results are in good quantitative agreement with experimental and simulation results reported in Pries et al. (1995) and Pries et al. (1998), respectively. Quantitative agreement with experimental results concerning other quantities such as the flow velocity is also obtained (see fig. 1b).

Another interesting feature of this optimal branching network is the branching exponent $\alpha(R, H)$. Experimentally, it is determined by averaging data collected from many junctions and many vascular generations (Frame and Sarelius, 1995). Doing this, for our optimal branching network, we obtain a figure very close to 3, i.e. Murray's law. However, Murray's law predicts a uniform value for the wall shear stress. 

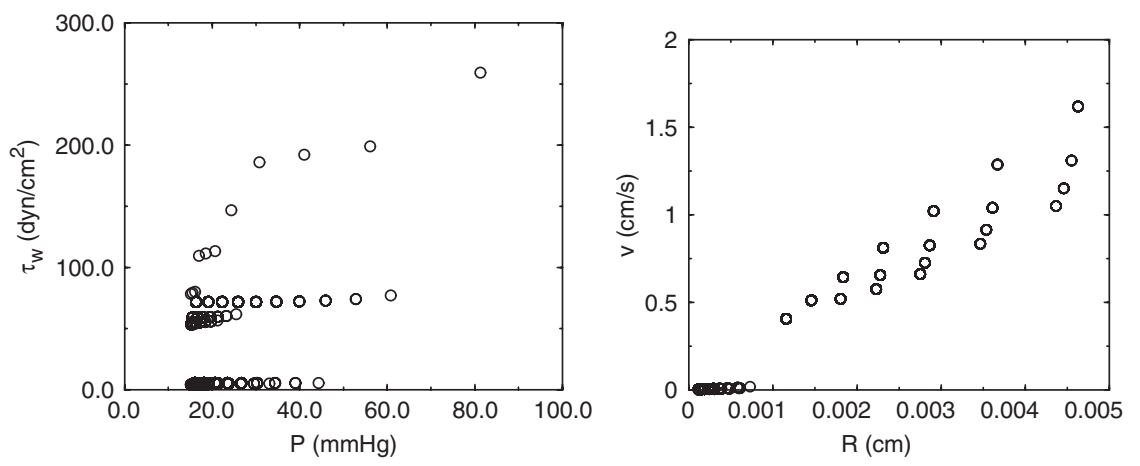

Fig. 1. Wall shear stress as a function of the pressure (a) and flow velocity as a function of the radius (b) in an optimal branching network. Our results are in quantitative agreement with simulation results reported by Pries et al. (1998) and experimental results obtained in Pries et al. (1995). See text for details.

This apparent contradiction has its origin in the use of a local bifurcation law (Equation (9)), rather than a global one with an average branching exponent. Karau et al. (2001) obtained similar results when studying vascular trees with heterogeneous branching exponents: even when the mean value of the branching exponent was three, non-uniform wall shear stress was observed. Their conclusion was that the influence on the wall shear stress in determining vessel radius is not necessarily manifested in the mean value of the branching exponent. This result also reconciles the experimental observations of a non-uniform wall shear stress with the geometrical design principle of space filling (Gafiychuk and Lubashevsky, 2001) and the supporting experimental evidence (Gazit et al., 1995).

\subsection{Vascular structural adaptation: coupling vascular structure to tissue metabolic demands}

The vasculature provides all the tissues in a particular organism with all the nutrients and metabolites they need to carry out their normal functions. As such, it cannot be a rigid, static structure. Rather, vascular networks are remarkably adaptable to local conditions, changing their structure in response to local increases in the demand for nutrients and other substances stimulated by increased activity.

As has been proposed in the previous section, vascular trees may comply with a design principle. Pries et al. (1998) proposed an adaptation mechanism to describe how different competing effects modify the lumen radius. One of these factors can be viewed as a hydrodynamic stimulus. It effects changes in the vessel radii such that the wall shear stress-pressure curve 
within the network approaches that reported in Pries et al. (1995). The second stimulus is a metabolic stimulus. It will cause an increase in the radii of the vessels in response to increasing metabolic demands of the surrounding tissue. Pries et al. (1998) argued that when the flux of haematocrit (given by $Q H$ ) transported through a vessel falls below a threshold value $\left(Q_{\text {ref }}\right)$, basically corresponding to the rate at which oxygen must be supplied to maintain homeostasis, its radius must be increased. The third, and last, stimulus that we consider is the so-called shrinking tendency. According to Pries et al. (1998), this stimulus corresponds to a natural tendency of the vessels to reduce their size in the absence of growth factors. This structural adaptation mechanism is mathematically implemented by:

$$
R(t+\Delta t)=R(t)+R \Delta t\left(\log \left(\frac{\tau_{\mathrm{w}}}{\tau(P)}\right)+k_{\mathrm{m}} \log \left(\frac{Q_{\mathrm{ref}}}{Q H}+1\right)-k_{\mathrm{s}}\right),
$$

where $\Delta t$ is the time step, $\tau(P)$ is the set-point value of the wall shear stress as a function of the pressure, $k_{\mathrm{m}}$ is a constant accounting for the strength of the metabolic stimulus. The first and second terms on the right-hand side of Equation (10) account for the hydrodynamic and metabolic stimuli, respectively. The constant $k_{\mathrm{s}}$ accounts for the shrinking tendency. For details of the actual expression for $\tau(P)$ we refer the reader to Pries et al. (1998).

We have incorporated complex blood rheology (by assuming that the viscosity depends on the radius and haematocrit in the manner described in the previous section) and structural adaptation in simulations of blood flow through a vascular network with a hexagonal structure (see fig. 2 and Alarcon et al., 2003). In these simulations, we assumed that initially all the vessels in the network had the same radii. We assumed further that the flow could be described by Poiseuille's law. Since, this assumption implies a linear relationship between flow rate and pressure drop between the ends of each vessel, we can also use Kirchoff's laws to calculate the flow rate and other hydrodynamic quantities of interest. The haematocrit in each vessel was calculated from an empirical law that states that at a bifurcation, the ratio of the haematocrits in each of the daughter vessels is proportional to the ratio of their flow rates. Furthermore, it has been found in model experiments with RBCshaped pellets that if the ratio of the flow rates exceeds some threshold value, then all the RBCs pass through the fastest branch (Fung, 1993). Once we know the haematocrit and the hydrodynamic quantities for each vessel, we apply the structural adaptation mechanism Equation (10). This procedure is repeated until the system reaches a stationary state (Alarcon et al., 2003). 


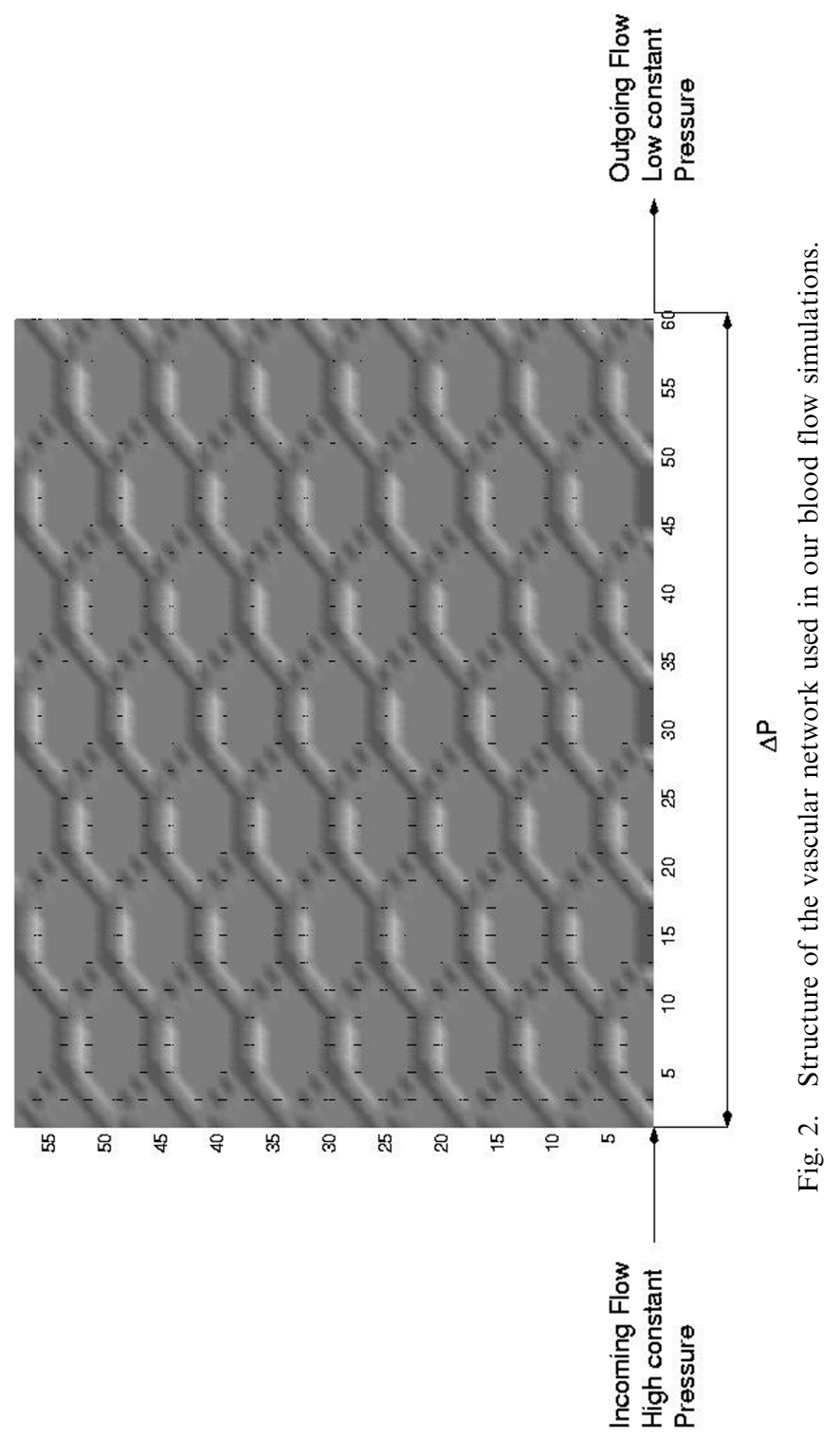


(a)

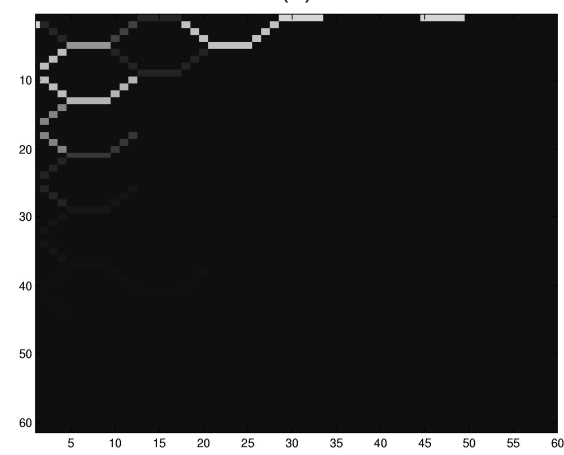

(b)

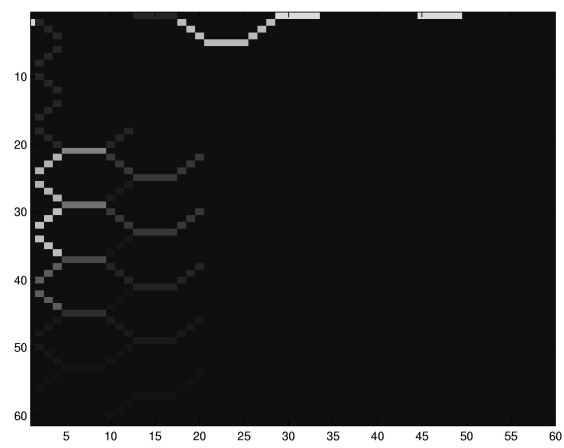

Fig. 3. Simulation results for the haematocrit distribution over a hexagonal vascular network. The results shown in (a) correspond to an uncoupled network (i.e. the structural adaptation of the vessels is carried out according to Equation (9), whereas (b) shows the results for a coupled network (vascular structural adaptation according to Equation (10)). The grey scale indicates the amount of haematocrit: lighter grey corresponds to higher haematocrit. See text for details.

The main result we obtain from our simulations (see fig. 3a) is that, as a result of the combination of complex blood rheology, structural adaptation, and uneven distribution of haematocrit at bifurcations, the distribution of both the blood flow and the haematocrit over the network are considerably heterogeneous.

The non-uniform haematocrit distribution may have important consequences for the dynamics of the surrounding tissue, since blood-borne oxygen is carried by the red blood cells. This has been studied in Alarcon et al. (2003), where the evolution of colonies of normal and cancer cells growing in response to the extracellular oxygen produced by the (inhomogeneous) distribution of haematocrit was investigated. Our simulations suggest that there may be significant differences between the behaviour of colonies growing under homogeneous and heterogeneous conditions.

The dynamics of the cell colony were modelled using a hybrid cellular automaton. Our two-dimensional model consisted of an array of $N \times N$ automaton elements, which will eventually be identified with real cells. The state of each element was defined by a state vector, whose components correspond to features of interest. To start with, the state vector had three components:

(i) occupation status, i.e. whether an element is occupied by a normal cell, a cancer cell, an empty space, or a vessel,

(ii) cell status, i.e. whether the cell is in a proliferative or quiescent state, and

(iii) the local oxygen concentration. 
The state vector evolved according to prescribed local rules that update a given element on the basis of its own state and those of its neighbours at the previous time step (Alarcon et al., 2003). While cells were considered as discrete entities, the oxygen concentration was treated as a continuous field, as the typical length of an oxygen molecule is very small compared to the characteristic size of a cell. The time evolution of the oxygen concentration was thus governed by a reaction-diffusion equation, with sinks, sources, and boundary conditions determined by the corresponding distribution of cells and haematocrit in the host vasculature.

The rules of the automaton were inspired by generic features of tumour growth, such as the ability of cancer cells to elude the control mechanisms, which maintain stasis in normal tissues. They can also alter their local environment, providing themselves with better conditions for growth and, eventually, for invasion of the host organism (King, 1996). In our simulations, we allowed the cancer cells to survive under lower levels of oxygen than normal cells. A well-known, though not exclusive, characteristic of cancer cells is their ability to enter a quiescent state, in which they suspend all activities (including cell division) that are not essential for their survival (Royds et al., 1998). They can remain in this latent state for a period of time, before starving to death. Finally, we incorporated into our model, competition between cancer and normal cells for existing resources, as proposed by Gatenby (1996). Normal tissue is a noncompetitive cell community, since, under conditions such as overcrowding or starvation, feedback mechanisms act to maintain tissue stasis. However, when members of this community become cancerous, a new population, with its own dynamics is formed. For further progression of the transformed population to occur, the tumour cells must compete for space and resources with the normal cells. For further details of our automaton rules, we refer the reader to Alarcon et al. (2003).

In simulations involving mixed colonies of normal and cancer cells (see fig. 4 for the initial conditions used in these simulations) we observed two distinct outcomes depending on whether we assumed the haematocrit distribution to be homogeneous or heterogeneous. When the haematocrit was distributed uniformly, the colony of cancer cells (which eventually eliminates the normal cell population) grew until it occupied all the available space (see fig. 5). Furthermore, the pattern of growth was isotropic. By contrast, when the haematocrit distribution was heterogeneous the colony saturated to a maximal size and its pattern of growth was anisotropic (see fig. 6).

The above behaviour can be explained as follows. If a cell divides and the new cell is situated in a poorly oxygenated region, it will die within a few iterations of the automaton. Consequently, all viable cells are located 


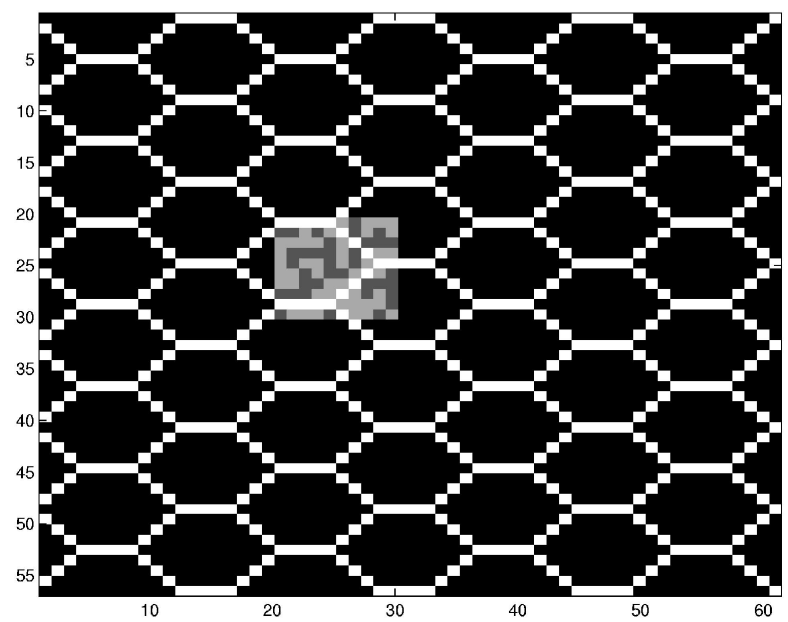

Fig. 4. Initial conditions for our growth simulations. The white spaces are occupied by vessels, light grey elements are occupied by cancer cells, dark grey elements correspond to normal cells, and black elements to empty spaces.

in well-oxygenated regions of the domain. While the regions in which cells accumulate are rich in oxygen, the amount of oxygen being supplied is finite and can only sustain a certain number of cells. The combination of these two effects with a non-uniform haematocrit distribution leads to the stationary patterns observed in figs. 5 and 6 (see also Alarcon et al., 2003).

One of the shortcomings of the model we have presented so far is that there is no feedback between the dynamics of the growing tissue and the adaptation of the vascular structure: the vasculature remains static while the colony develops. In practice, the vasculature remodels itself according to the local needs of the surrounding tissue either via dilation of the lumen (structural adaptation of the radius) as occurs, for example during the inflamatory response, or via angiogenesis, as in the cases of wound healing and tumour growth.

As a first attempt at developing a more realistic model, we have introduced into our basic model (Alarcon et al., 2005b) internal dynamics for each of the automaton elements that may occupy a cell. These dynamics account for three different intracellular processes, namely the cell cycle, apoptosis, and secretion of vascular endothelial growth factor (VEGF), a potent angiogenic factor. For details, we refer the reader to Alarcon et al. (2005b). In brief, we assume that when subjected to oxygen starvation (i.e. hypoxia) both cancer and normal cells produce and then release VEGF. Released VEGF diffuses through the tissue and eventually reaches the vessels. There it activates the cells' proteolytic machinery, causing the basal 


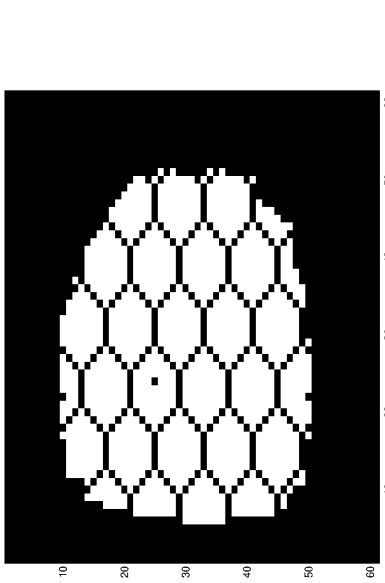

흠

焉.

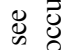

๘ี

$\sum \stackrel{0}{=}$

$\stackrel{\dot{\vec{\theta}}}{\vec{z}}$

劳

$\frac{7}{3}$

这

速它它

जै के की

害商

ठํㅠ

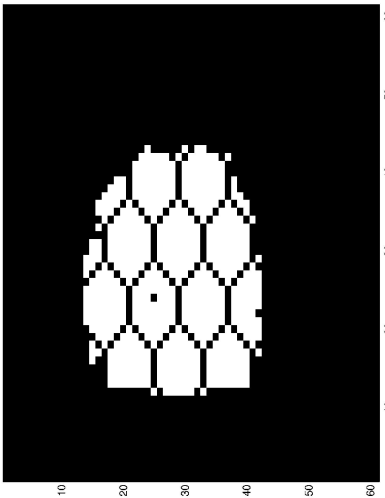

告䑰

웡

${ }_{0} 3$

워

过

बे 0

d)

$\Xi$ 믐

卷 $=$

ষ্ট

芯管

प०

त 00

응 흥

फ

$0 \pm \frac{1}{0}$

$\Xi \Xi$

뭉

छ.

छ융

풍

응

응

핀

范.

क

ญ्.

F

is

证䒕 


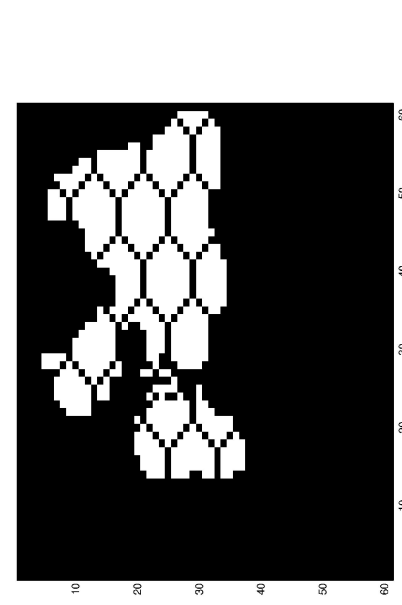

:

它

疍

$\Xi \dot{s}$

कon के

:

क्षे

ठ

结

a

을 짐

킁융

0 을

竞 票

包

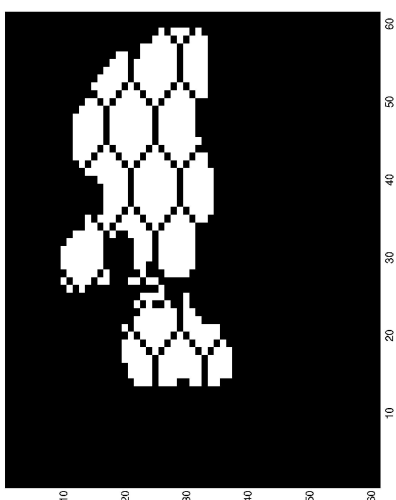

흘

论

离量

$\Xi$ 政

고ㅇㅝㅠ

उद के

氖言

ठ० क

¿ 5

ते

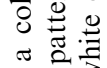

403

표

으 핱

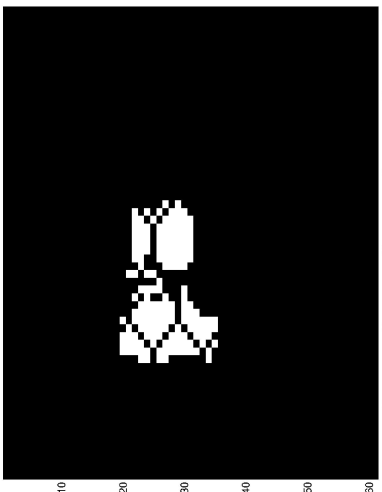

卷

ठ है है

$\Xi 3$

0

茟

용. 욜

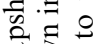

范

क

.

F.

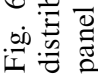


membrane of the vessels to be degraded. This increases leakiness of the vessel and enables the endothelial cells to migrate towards the source of VEGF. Rather than modelling tumour-induced angiogenesis in all its complexity, we have assumed that the angiogenic response in a given region can be described in an effective manner by an increase in the radius of the vessels in that region. (Similar approximations are common in the Plant Sciences, where the complex branching structure of the roots is replaced by a cylindrical tube of an equivalent radius.) In our model, growth of a vessel radius is thus indirectly stimulated by hypoxia, and directly stimulated by VEGF. The model we propose to account for the interactions between the vasculature and the underlying tissue involves modifying Equation (10) in the following way:

$$
\begin{aligned}
R(t+\Delta t)= & R(t)+R \Delta t\left(\log \left(\frac{\tau_{\mathrm{w}}}{\tau(P)}\right)\right. \\
& \left.+k_{\mathrm{m}}\left(1+\frac{V}{V_{0}+V}\right) \log \left(\frac{Q_{\mathrm{ref}}}{Q H}+1\right)-k_{\mathrm{s}}\right),
\end{aligned}
$$

where $V$ is the (local) concentration of VEGF. Equation (11) implies that the metabolic stimulus will be more intense for vessels in hypoxic regions, since in these regions rates of VEGF secretion will be higher.

The effect that this modification to the structural adaptation algorithm has on the distribution of haematocrit is shown in fig. $3 \mathrm{~b}$. When the effect of VEGF is introduced, vessels in hypoxic regions tend to grow larger under the angiogenic stimulus of VEGF. As a result blood flow through those vessels increases, and therefore more haematocrit is supplied. The net effect of this is a more effective distribution of haematocrit: the red blood cells are more evenly distributed than when the evolution of the vasculature is independent of the surrounding tissue. This effect can be seen by comparing fig. $3 \mathrm{a}$ (uncoupled case) and fig. 3b (coupled case). As a consequence, we have shown in Alarcon et al. (2005b) that a colony that is perfused by a vasculature whose evolution is influenced by the colony grows to an equilibrium size that is bigger than that obtained when there is no coupling.

\section{CONCLUSIONS}

The main aim of the research summarised in this chapter is to make clear that accounting for the properties of the vascular system and blood flow is necessary to develop realistic models of angiogenesis. Most of the models currently in the literature concentrate on endothelial cell migration in response to the chemotactic stimulus of the VEGF, and neglect most of 
the aforementioned issues (i.e. blood flow and haematocrit heterogeneity, coupling between metabolic needs of tissue and vascular structural adaptation). However, these phenomena must be taken into account when coupling models of angiogenesis to models of solid tumour growth. The usual assumption is that the local concentration of oxygen or other nutrients is proportional to the local concentration of endothelial cells. We have shown that this assumption may be overly simplistic due to blood flow heterogeneity. In particular, we have shown that when the nutrient concentration is proportional to the local endothelial cell concentration then the growth of the colony is markedly different from that for which blood flow heterogeneity is taken into account (compare figs. 5 and 6).

A similar situation arises when modelling chemotherapy. If we assume that the blood-borne concentration of the drug is proportional to the local concentration of endothelial cells, we might overestimate the concentration of drug reaching regions in which blood flow is poor and underestimate the concentration in regions where blood flow is high. As a result, the intrinsic non-uniformity in the spatial structure of many solid tumours may be an important factor in predicting the efficacy of chemotherapy protocols (see Riba et al., 2003).

\section{REFERENCES}

Alarcon, T., Byrne, H.M., Maini, P.K., 2003. A cellular automaton model for tumour growth in inhomogeneous environment. J. Theor. Biol. 225, 257-274.

Alarcon, T., Byrne, H.M., Maini, P.K., 2005a. A design principle for vascular beds: the effects of complex blood rheology. Microvasc. Res. 69, 156-172.

Alarcon, T., Byrne, H.M., Maini, P.K., 2005b. A multiple scale model of tumour growth. Multiscale Model Sim. 3, 440-475.

Anderson, A.R.A., Chaplain, M.A.J., 1998. Continuous and discrete mathematical models of tumour-induced angiogenesis. Bull. Math. Biol. 60, 857-899.

Anderson, A.R.A., Chaplain, M.A.J., 1998. A mathematical model for capillary network formation in the absence of endothelial cell proliferation. Appl. Math. Lett. 11, 109-114.

Balding, D., McElwain, D.L.S., 1985. A mathematical model of tumour-induced capillary growth. J. Theor. Biol. 114, 53-73.

Breward, C.J.W., Byrne, H.M., Lewis, C.E., 2003. A multiphase model describing vascular tumour growth. J. Math. Biol. 65, 609-640.

Byrne, H.M., Chaplain, M.A.J., 1995. Mathematical models for tumour angiogenesis: numerical simulations and nonlinear wave solutions. Bull. Math. Biol. 57, 461-486.

Edelstein, L., 1982. The propagation of fungal colonies: a model of tissue growth. J. Theor. Biol. 98, 679-701.

Folkman, J., 1995. Angiogenesis in cancer, vascular, rheumatoid, and other disease. Nature Med. 1, 27-31.

Frame, M.D.S., Sarelius, I.H., 1995. Energy optimisation and bifurcation angles in the microcirculation. Microvasc. Res. 50, 301-310. 
Fung, Y.-C., 1993. Biomechanics. Springer, New York.

Gafiychuk, V.V., Lubashevsky, I.A., 2001. On the principles of the vascular network branching. J. Theor. Biol. 212, 1-9.

Gatenby, R.A., 1996. Application of competition theory to tumour growth: implications for tumour biology and treatment. Eur. J. Cancer. 32A, 722-726.

Gazit, Y., Berk, D.A., Leunig, M., Baxter, L.T., Jain, R.K., 1995. Scale-invariant behaviour and vascular network formation in normal and tumour tissue. Phys. Rev. Lett. $75,2428-2431$.

Jain, R.K., 2001. Delivery of molecular and cellular medicine to solid tumours. Adv. Drug Delivery Rev. 46, 146-168.

Karau, K.L., Krenz, G.S., Dawson, C.A., 2001. Branching exponent heterogeneity and wall shear stress distribution in vascular trees. Am. J. Phisol. 280, H1256-H1263.

King, R.J.B., 1996. Cancer Biology. Longman, Harlow.

Kurz, H., Sandau, K., 1997. Modelling of blood vessel development - bifurcation pattern and hemodynamics, optimality and allometry. Comments Theor. Biol. 4, 261-291.

LaBarbera, M., 1990. Principles of design of fluid-transport systems in Zoology. Science 249, 992.

Levine, H., Pamuk, S., Sleeman, B.D., Nilsen-Hamilton, M., 2001. Mathematical modelling of capillary formation and development in tumour angiogenesis: penetration into the stroma. Bull. Math. Biol. 63, 801-863.

Mantzaris, N., Webb, S., Othmer, H.G., 2004. Mathematical modelling of tumour-induced angiogenesis. J. Math. Biol. 49, 111-187.

Murray, C.D., 1926. The physiological principle of minimum work, I: the vascular system and the cost of volume. Proc. Natl. Acad. Sci. USA 12, 207-214.

Muthukkaruppan, V.R., Kubai, L., Auerbach, R., 1982. Tumour-induced neovascularisation in the mouse eye. J. Natl. Cancer Inst. 69, 699-705.

Orme, M.E., Chaplain, M.A.J., 1996. A mathematical model of the first steps of tumour-related angiogenesis: capillary sprout formation and secondary branching. IMA J. Math. Appl. Med. Biol. 13, 73-98.

Orme, M.E., Chaplain, M.A.J., 1997. Two-dimensional models of tumour angiogenesis and anti-angiogenesis strategies. IMA J. Math. Appl. Med. Biol. 14, 189-205.

Othmer, H.G., Stevens, A., 1997. Aggregation, blow-up and collapse: the ABC's of taxis in reinforced random walks. SIAM J. Appl. Math. 57, 1044-1081.

Panovska, J., 2004. Mathematical modelling of tumour growth and application to therapy. DPhil Thesis. Oxford. In preparation.

Preziosi, L., 2003. Cancer modelling and simulation. Chapman and Hall/CRC.

Pries, A.R., Secomb, T.W., Gessner, T., Sperandio, M.B., Gross, J.F., Gaehtgens, P., 1994. Resistance to blood flow in microvessels in vivo. Circ. Res. 75, 904-915.

Pries, A.R., Secomb, T.W., Gaehtgens, P., 1995. Design principles of vascular beds. Circ. Res. 77, 1017-1023.

Pries, A.R., Secomb, T.W., Gaehtgens, P., 1998. Structural adaptation and stability of microvascular networks: theory and simulations. Am. J. Physiol. 275, H349-H360.

Riba, B., Marron, K., Agur, Z., Alarcon, T., Maini, P.K., 2003. A mathematical model of Doxorubicin treatment efficiency in non-Hodgkin's lymphoma: investigation of the current protocol through theoretical modelling results. Bull. Math. Biol. 67, 79-99.

Royds, J.A., Dower, S.K., Qwarnstrom, E.E., Lewis, C.E., 1998. Response of tumour cells to hypoxia: role of p53 and NFkB. J. Clin. Pathol. Mol. Pathol. 51, 55-61. 
